\title{
Decomposição da fitomassa de plantas de cobertura e liberação de nitrogênio em função da quantidade de resíduos aportada ao solo sob sistema plantio direto
}

\author{
Fitomass decomposition and nitrogen release of cover crops in \\ function of the level of residue input to soil under no-tillage system \\ José Alan de Almeida Acosta ${ }^{\mathrm{I}}$ Telmo Jorge Carneiro Amado ${ }^{{ }^{*}}$ \\ Leandro Souza da Silva ${ }^{\mathrm{I}}$ Anderson SantiI Mirla Andrade Weber ${ }^{\mathrm{II}}$
}

RESUMO

\begin{abstract}
O sucesso do sistema plantio direto (SPD) está alicerçado na rotação de culturas, que proporciona elevada adição de diferentes tipos de resíduos culturais ao solo. Entretanto, cada resíduo cultural tem uma cinética específica de decomposição, o que é determinante na mineralização e imobilização líquida de nitrogênio $(N)$ no solo. Este trabalho teve como objetivo avaliar a taxa de decomposição e a liberação de $N$ de plantas de cobertura de inverno, quando diferentes quantidades de fitomassa da parte aérea foram aportadas à superfície do solo. O experimento foi conduzido em Argissolo arênico na área do Departamento de Solos da Universidade Federal de Santa Maria (UFSM), com delineamento experimental de blocos ao acaso, com três repetições, durante as safras de 2003/04 e 2004/05. Avaliou-se a decomposição e liberação de nitrogênio $(N)$ dos resíduos de aveia preta, nabo forrageiro e ervilhaca através de bolsas de decomposição contendo quantidades equivalentes a 3, 6 e $9 \mathrm{Mg} \mathrm{ha}^{-1}$ de matéria seca e dispostos em parcelas com $80 \mathrm{~m}^{2}$, contendo os mesmos níveis de resíduos das bolsas. As coletas das bolsas foram realizadas aos 7, 14, 28, 42, 63, 84, 113 e 141 dias após o manejo das plantas de cobertura. As quantidades de resíduos aportadas não influenciaram as taxas de decomposição. Entretanto, os processos de mineralização e imobilização de $N$ foram afetados pelo nível de aporte de resíduos e governados, principalmente, pela relação $C / N$. A maior taxa de decomposição dos resíduos e a maior liberação de $N$ seguiram a ordem: ervilhaca, nabo forrageiro e aveia preta. A relação $C / N$ dos resíduos influenciou a magnitude dos compartimentos lábil e recalcitrante da fitomassa das plantas de cobertura.
\end{abstract}

Palavras-chave: adubação verde, mineralização de $N$, relação $C / N$, imobilização de $N$.

\section{ABSTRACT}

The no-tillage system success relies on higher addition of different types of crop residues to the soil, each one with specific decomposition kinetics which drives the net mineralization and immobilization of the nitrogen $(N)$ in the soil. This manuscript had as objective to evaluate the decomposition rate and $N$ released from winter cover crops residues under different above ground biomass input to the soil. The experiment was carried out in the experimental area of the Soil Department at Federal University of Santa Maria, in random complete block design, with three replications, during 2003/04 and 2004/05. The decomposition and the $N$ released from black oat, oil radish and hairy vetch residues were investigated using mesh bags of decomposition containing fitomass equivalent to 3, 6 and $9 \mathrm{Mg}$ of dry matter ha $\mathrm{a}^{-1}$ placed in plots with $80 \mathrm{~m}^{2}$ with the same level of crop residues used in the mesh bags. They were collected at 7, 14, 28, 42, 63, 84, 113 and 141 days after cover crops management. The amount of residues input did not influence the decomposition rate. However, the $N$ mineralization and immobilization processes were affected by the level of residues input and they were driven by $\mathrm{C} / \mathrm{N}$ ratio. The larger decomposition residues rate and higher $N$ release followed the order: vetch, radish oil and black oat. The $\mathrm{C} / \mathrm{N}$ ratio drives the size of labil and recalcitrant compartiments of cover crops fitomass.

Key words: green manure; $N$ mineralization; $C / N$ ratio; $N$ immobilization.

\section{INTRODUÇÃO}

A decomposição dos resíduos culturais aportados ao solo é realizada, essencialmente, pelos microrganismos heterotróficos que, nesse processo, obtêm elementos essenciais para a sua nutrição e o carbono necessário à produção de energia e formação de tecidos microbianos (AITA, 1997). Porém, a velocidade

\footnotetext{
IDepartamento de Solos, Centro de Ciências Rurais (CCR), Universidade Federal de Santa Maria (UFSM), 97105-900, Santa Maria, RS, Brasil. E-mail: telmo.amado@pq.cnpq.br.*Autor para correspondência.

IICentro Nacional de Pesquisa de Trigo, Empresa Brasileira de Pesquisa Agropecuária (EMBRAPA), Passo Fundo, RS, Brasil.

IIIFaculdade de Agronomia, Universidade Federal do Pampa (UNIPAMPA), Bagé, RS, Brasil.
} 
deste processo é determinada por fatores bióticos e abióticos que definem a persistência dos resíduos na superfície do solo (ESPÍNDOLA et al., 2006).

Entre os fatores que afetam a taxa de decomposição de resíduos estão o contato solo/ resíduo (AMADO et al., 2000; CARVALHO et al., 2008), o regime hídrico (PARTON et al., 2007), a adição de N inorgânico ao solo (MARY et al., 1996), a temperatura do solo (KOENIG et al., 1994), a relação C/N (MARY et al., 1996; CARVALHO et al., 2008; TORRES \& PEREIRA, 2008), o teor de lignina e polifenóis (KOENIG et al., 1994; ESPÍNDOLA et al., 2006) e a concentração de $\mathrm{N}$ no tecido vegetal (PARTON et al., 2007; MEDRADO et al., 2011). No entanto, entre as características qualitativas, a relação $\mathrm{C} / \mathrm{N}$ dos resíduos aportados ao solo assume papel preponderante na decomposição e na relação entre mineralização e imobilização de $\mathrm{N}$ pela biomassa microbiana. JANSSEN (1996), analisando vários estudos relacionados à mineralização do $\mathrm{N}$, sustentou que a fração de $\mathrm{N}$ orgânico mineralizada está inversamente relacionada à relação $\mathrm{C} / \mathrm{N}$, de maneira idêntica à decomposição dos resíduos. Na literatura, são encontrados diversos valores de relação $\mathrm{C} / \mathrm{N}$, a partir dos quais ocorre imobilização líquida de $\mathrm{N}$ (KUMAR \& GOH, 2003). Contudo, estes variam com o tipo de planta e o estádio de maturação que estas são adicionadas ao solo (MONTEIRO et al., 2002).

Geralmente, resíduos vegetais com relação $\mathrm{C} / \mathrm{N}$ de 25 causam equilíbrio entre os processos de imobilização e mineralização. Valores superiores causam imobilização líquida, enquanto que valores inferiores promovem mineralização líquida de $\mathrm{N}$ ao solo (AITA, 1997). KUDEYAROV (1999) também demonstrou que a imobilização ocorreu quando a relação $\mathrm{C} / \mathrm{N}$ foi maior que 25. Entretanto, JENSEN (1997), trabalhando com resíduos de lentilha, encontrou imobilização líquida nos períodos iniciais de decomposição, quando a relação $\mathrm{C} / \mathrm{N}$ era de 15 . Este trabalho indica que a imobilização pode ocorrer em diversas fases da decomposição dos resíduos.

Em manejos conservacionistas, sob clima tropical e subtropical, preconiza-se a adição de elevadas quantidades de resíduos culturais, compensando a rápida decomposição, a fim de manter a superfície do solo protegida pelo maior período de tempo possível (DERPSCH et al., 2010) e para incrementar o teor de matéria orgânica do solo (AMADO et al., 2006). Para as leguminosas, utilizadas como plantas de cobertura, o elevado aporte de fitomassa é requerido visando à maior disponibilidade de $\mathrm{N}$ às culturas cultivadas em sucessão (AMADO et al., 2002). No entanto, para a planta de cobertura atender a demanda em $\mathrm{N}$ da cultura subsequente, há a necessidade de que a liberação de $\mathrm{N}$ dos resíduos aportados ocorra em sincronismo com a demanda da cultura em sucessão (AMADO et al., 2000; AITA \& GIACOMINI, 2003). Dessa forma, justifica-se que a quantidade de resíduos aportada à superfície do solo pelas diferentes plantas de cobertura seja investigada quanto a sua influência na taxa de decomposição e na liberação do $\mathrm{N}$ mineralizado. Nesse contexto, este trabalho teve como principal objetivo avaliar a dinâmica de decomposição e liberação de $\mathrm{N}$ em função da quantidade de resíduos aportados ao solo pelas principais plantas de cobertura de inverno utilizadas no SPD do Sul do Brasil.

\section{MATERIAL E MÉTODOS}

O experimento foi realizado na área do Departamento de Solos da Universidade Federal de Santa Maria (UFSM), Santa Maria, RS, em um solo classificado como um ARGISSOLO VERMELHO Distrófico arênico (EMBRAPA, 2006). O trabalho foi conduzido com delineamento em blocos ao acaso, com três repetições, durante o desenvolvimento da cultura do milho das safras 2003/04 e 2004/05. O clima da região é do tipo “Cfa2”, de acordo com a classificação climática de Köppen, com precipitação média anual de $1.686 \mathrm{~mm}$ e temperatura média anual de $19,3^{\circ} \mathrm{C}$, variando entre $9,3^{\circ} \mathrm{C}$ (média do mês mais frio) e $31,5^{\circ} \mathrm{C}$ (média do mês mais quente). Os dados de temperatura e precipitação média mensal foram obtidos na estação meteorológica da UFSM, distante 1,5km da área experimental.

A dinâmica de decomposição e a liberação de $\mathrm{N}$ de resíduos culturais de aveia preta (Avena strigosa Schreb), ervilhaca peluda (Vicia villosa Roth) e nabo forrageiro (Raphanus sativus L.) foram avaliadas através de bolsas de decomposição confeccionadas em náilon, com malha de $0,5 \mathrm{~mm}$ e dimensões externas de $0,18 \times 0,19 \mathrm{~m}$. As bolsas foram preenchidas com quantidades equivalentes a 3, 6 e $9 \mathrm{Mg}$ ha $^{-1}$ de matéria seca (MS). Os resíduos apresentaram, respectivamente, em 2003 e 2004, relações C/N de 11 e 16 nos resíduos de ervilhaca, 44 e 39 nos resíduos de aveia preta e valores intermediários de 32 e 26 nos resíduos de nabo forrageiro.

Imediatamente após o manejo das culturas de inverno, as bolsas de decomposição foram dispostas a campo, em parcelas, com dimensões de 5x16m, com quantidades de resíduo semelhantes a dos níveis de resíduos investigados, visando representar as condições microclimáticas de umidade e temperatura. Os ajustes dos níveis de resíduos nas parcelas foram 
feitos por remoção e adição de resíduos, seguidos de sucessivas pesagens. Assim, para o nível de $3 \mathrm{Mg}$ $\mathrm{ha}^{-1}$, houve necessidade de remoção de resíduos das parcelas, que foram transferidos para o nível de $9 \mathrm{Mg} \mathrm{ha}{ }^{-1}$, no qual ainda foram adicionados resíduos provenientes de áreas adjacentes. Cada nível de resíduo recebeu 24 sacos de decomposição (8 em cada bloco), distribuídos paralelamente às linhas de milho em posição central da entrelinha. As coletas foram realizadas aos 7, 14, 28, 42, 63, 84, 113 e 141 dias (DAM), após sua colocação no campo, em ambos os anos avaliados, com três repetições por data de coleta. Após cada coleta, os resíduos foram secos em estufa a $65^{\circ} \mathrm{C}$ até peso constante, para a determinação da MS e do $\mathrm{N}$ remanescente. $\mathrm{O}$ preparo das amostras seguiu a metodologia descrita em AMADO et al. (2003). Para a determinação da quantidade de $\mathrm{N}$, seguiu-se a metodologia descrita por TEDESCO et al. (1995).

Para descrever a decomposição dos resíduos, aplicou-se o modelo de decaimento exponencial duplo, descrito por PLANTAE \& PARTON (2007): $P_{t}=A e^{(-k . t)}+B e^{(-k . t)}$, em que: $P_{t}$ é o percentual do resíduo existente no tempo $t$; $A$ é a proporção do compartimento de resíduo lábil; $B$ é a proporção do compartimento de resíduo recalcitrante; $k_{a} e k_{b}$ são constantes de decomposição de cada compartimento. A partir dos valores da constante de decomposição da MS, estimou-se o tempo de meiavida $\left(t_{1 / 2}=0,693 / \mathrm{k}\right)$ de cada compartimento e o tempo necessário para decompor a metade da quantidade dos resíduos aportados ( $\left.\mathrm{t}_{50 \%}\right)$.

Os resultados obtidos foram submetidos à análise da variância. Para os fatores de natureza qualitativa, as médias de tratamentos foram comparadas através do teste de Duncan $(\mathrm{P}<0,05)$. Os dados referentes à taxa de decomposição foram ajustados por modelos não lineares de regressão para cada ano de estudo (2003 e 2004) e cada data foi comparada através da Diferença Mínima Significativa (DMS) pelo teste Tukey $(\mathrm{P}<0,05)$, através do software SAS 5.0 (2004).

\section{RESULTADOS E DISCUSSÃO}

Durante o período de outubro a março, no qual foi conduzido o experimento, a temperatura média de cada mês foi semelhante entre os anos investigados, porém a precipitação média mensal foi distinta, totalizando 511mm a mais em 2003 do que em 2004 (Figura 1). Ainda, as maiores diferenças na precipitação observadas entre os anos foram verificadas nos três primeiros meses do período experimental.

A decomposição dos resíduos foi favorecida pelos regimes de temperatura e precipitação ocorridos durante o período experimental. Assim, a temperatura média, que variou de 20 a $25^{\circ} \mathrm{C}$ e as frequentes precipitações favoreceram a decomposição dos resíduos (ESPÍNDOLA et al., 2006; CARVALHO et al., 2008). O maior volume de precipitação em 2003 proporcionou, independente do tipo de resíduo, uma maior taxa de decomposição em relação a 2004. As curvas de decomposição dos resíduos apresentaram padrões distintos na cinética de decomposição, que variou de acordo com o tipo de resíduo, principalmente

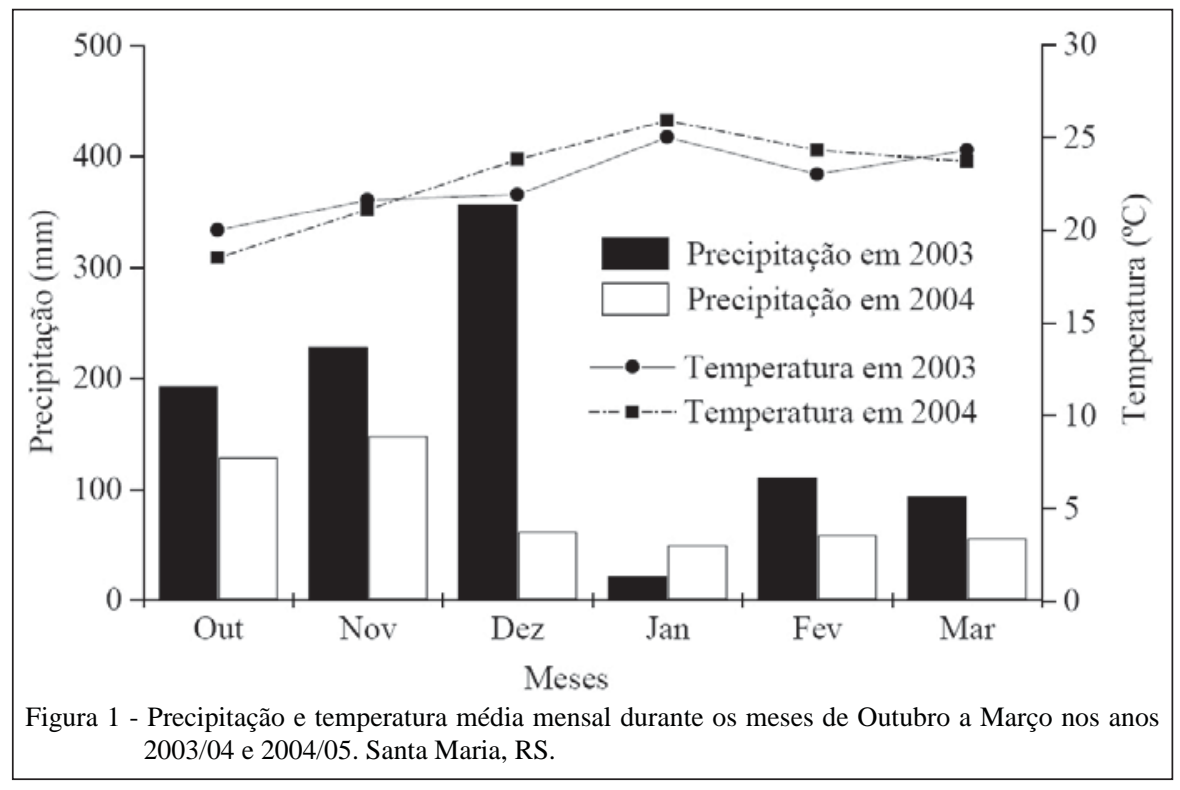

Ciência Rural, v.44, n.5, mai, 2014. 
na fase inicial de decomposição, sendo influenciada pelo conteúdo de $\mathrm{N}$ e pela relação $\mathrm{C} / \mathrm{N}$ (TORRES \& PEREIRA, 2008; MEDRADO et al., 2011). O maior conteúdo de $\mathrm{N}$ e a menor relação $\mathrm{C} / \mathrm{N}$ na ervilhaca, conforme esperado, proporcionaram uma maior taxa de decomposição dos resíduos (Figura 2).
Nos dois anos do estudo, a ervilhaca sempre apresentou a maior taxa de decomposição, seguida do nabo forrageiro e da aveia preta, que decompôs menos de $50 \%$ de seus resíduos, mesmo transcorridos cinco meses após o manejo. CERETTA et al. (2002); AITA \& GIACOMINI (2003); e AMADO et al. (2003), que
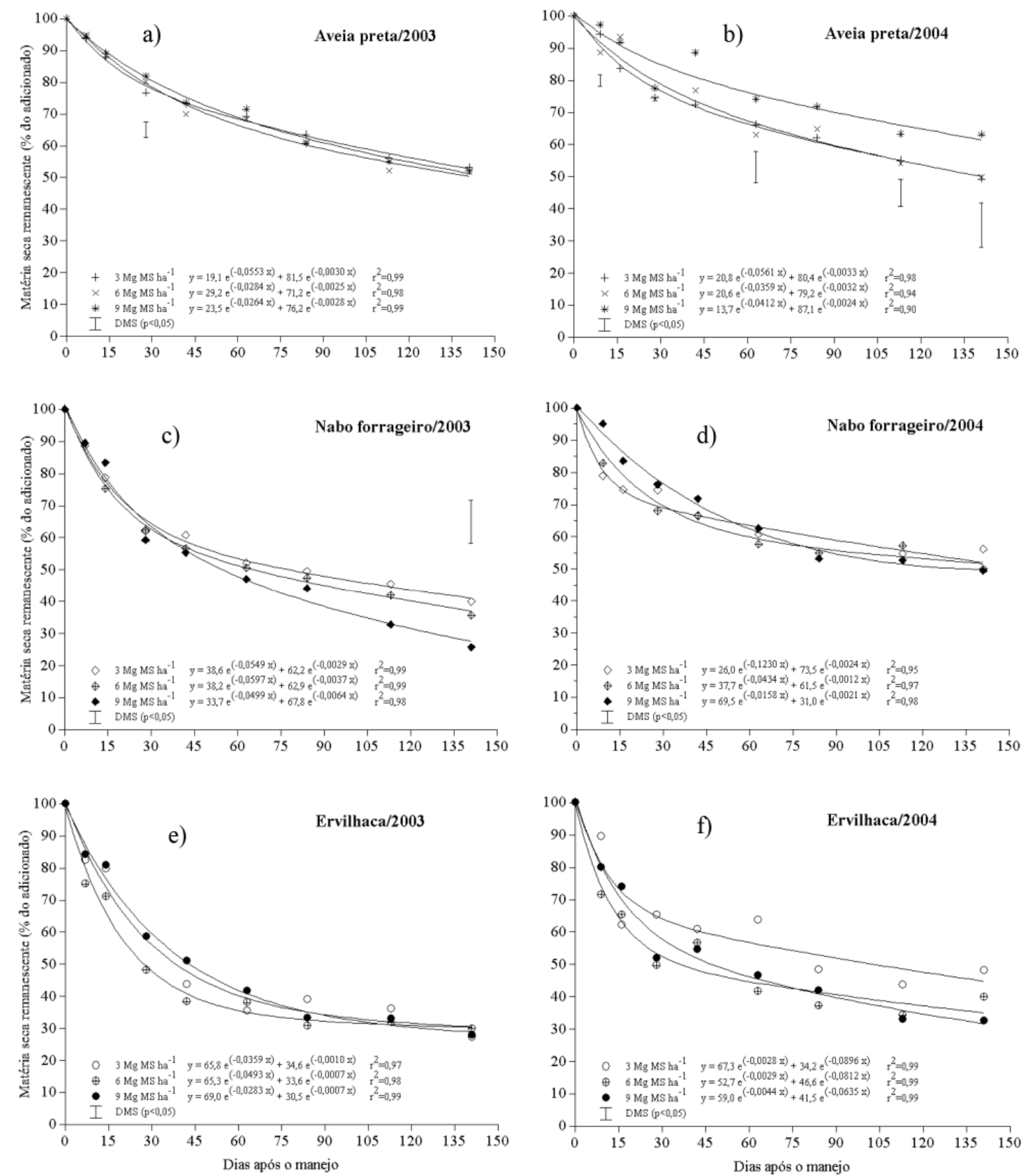

Figura 2 - Curvas de decomposição dos resíduos de aveia preta, nabo forrageiro e ervilhaca em função da quantidade de resíduos aportada ao solo. As barras verticais indicam a Diferença Mínima Significativa (DMS) pelo teste Tukey $(\mathrm{P}<0,05)$. 
anteriormente conduziram estudos dessa natureza e obtiveram resultados semelhantes, creditaram as diferenças entre taxas de decomposição à distinta relação $\mathrm{C} / \mathrm{N}$.

A taxa de decomposição, independente do tipo de resíduo, não foi influenciada pela quantidade de resíduos culturais adicionados ao solo (Figura 2; Tabela 1). A aveia preta foi a única planta de cobertura que, em 2004, apresentou em torno dos 60 DAM, uma menor taxa de decomposição no maior nível de resíduo aportado. A ausência de efeito da quantidade de resíduos aportados sob SPD na taxa de decomposição não era esperada. Esse fato pode estar associado a uma interação de fatores, tais como: a) às condições de temperatura e precipitação favoráveis à atividade biológica durante o período experimental (Figura 1) e, consequentemente, a decomposição dos resíduos; b) à elevada capacidade dos microrganismos em aumentar sua população, em resposta à maior oferta de carbono orgânico (ROBERTSON \& GROFFMAN, 2007), a maior quantidade de $\mathrm{N}$ na fitomassa aportada (MARY et al., 1996) e o incremento do teor de $\mathrm{N}$ no solo manejado sob SPD (AMADO et al., 2000). Esses fatores foram preponderantes em relação ao efeito de redução da temperatura do solo proporcionada pelo maior aporte de resíduos vegetais na superfície do solo (AMADO et al., 1990) e ao menor contato dos resíduos com o solo, devido à sobreposição de peças de resíduos. Assim, a temperatura média do ar em torno de $23^{\circ} \mathrm{C}$ (Figura 1) determinou que, no maior nível de resíduos, a provável redução de alguns graus centígrados na temperatura do solo não fosse limitante à atividade biológica. Anteriormente, CARVALHO et al. (2008), avaliando taxa de decomposição de diferentes tipos de resíduos, reportaram que, nos primeiros 6 meses, a incorporação de resíduos de leguminosas com baixa relação C/N não incrementou a taxa de decomposição em relação à manutenção dos resíduos na superfície.

A liberação de $\mathrm{N}$ dos resíduos foi influenciada pela quantidade e tipo de resíduos adicionados ao solo (Figura 3). Observou-se que a interação entre a quantidade e o tipo dos resíduos determinou a intensidade dos processos de mineralização/imobilização de $\mathrm{N}$ de cada planta de cobertura. Assim, os resíduos de ervilhaca, com elevado conteúdo de $\mathrm{N}$ e baixa relação $\mathrm{C} / \mathrm{N}$, não apresentaram imobilização líquida e liberaram até $50 \%$ do $\mathrm{N}$ acumulado na fitomassa nos primeiros 30 dias (Figura 3e e 3f), podendo fornecer ao solo quantidades superiores a $100 \mathrm{~kg} \mathrm{ha}^{-1}$ de N (ACOSTA et al., 2011). Este efeito foi observado em todas as quantidades de fitomassa aportadas, sendo que a quantidade de $9 \mathrm{Mg} \mathrm{ha}^{-1}$, no ano de 2003, apresentou

Tabela 1 - Proporção do compartimento lábil (A), recalcitrante (B), constantes de decomposição ( $\mathrm{k}_{\mathrm{a}}$ e $\mathrm{k}_{\mathrm{b}}$ ), tempo de meia-vida ( $\mathrm{t}_{1 / 2}$ ) de cada compartimento e tempo necessário para decompor $50 \%\left(\mathrm{t}_{50 \%}\right)$ dos resíduos das plantas de cobertura dentro de cada nível de resíduo avaliado. Médias de 2003 e 2004.

\begin{tabular}{|c|c|c|c|c|c|c|c|c|c|}
\hline $\begin{array}{l}\text { Plantas de } \\
\text { cobertura }\end{array}$ & $\mathrm{NR}^{1}$ & A \% & $\mathrm{k}_{\mathrm{a}} \mathrm{g} \mathrm{g}^{-1} \mathrm{dia}^{-1}$ & $\mathrm{t}_{1 / 2}$ dia & В \% & $\mathrm{k}_{\mathrm{b}} \mathrm{g} \mathrm{g^{-1 }} \mathrm{dia}^{-1}$ & $\mathrm{t}_{1 / 2}$ dia & $\mathrm{t}_{50 \%}$ dia & $r^{2}$ \\
\hline \multirow{4}{*}{ Aveia preta } & 3 & 20,0 & $-0,0557$ & 12 & 81,0 & $-0,0032$ & 214 & 147 & 0,991 \\
\hline & 6 & 24,9 & $-0,0322$ & 22 & 75,2 & $-0,0029$ & 235 & 141 & 0,969 \\
\hline & 9 & 18,6 & $-0,0339$ & 20 & 81,7 & $-0,0027$ & 258 & 203 & 0,945 \\
\hline & Média & $21,2 b^{2}$ & $-0,0406$ a & $18 \mathrm{a}$ & 79,3 а & $-0,0030$ a & $236 \mathrm{a}$ & $164 \mathrm{a}$ & \\
\hline \multirow{4}{*}{$\begin{array}{l}\text { Nabo } \\
\text { forrageiro }\end{array}$} & 3 & 32,4 & $-0,0889$ & 8 & 67,9 & $-0,0027$ & 257 & 115 & 0,975 \\
\hline & 6 & 38,0 & $-0,0515$ & 13 & 62,3 & $-0,0025$ & 277 & 113 & 0,983 \\
\hline & 9 & 51,6 & $-0,0329$ & 21 & 49,9 & $-0,0042$ & 164 & 90 & 0,985 \\
\hline & Média & $40,7 \mathrm{a}$ & $-0,0578$ a & $14 \mathrm{a}$ & $60,0 \mathrm{~b}$ & $-0,0031 \mathrm{a}$ & $233 \mathrm{a}$ & $106 \mathrm{~b}$ & \\
\hline \multirow{4}{*}{ Ervilhaca } & 3 & 50,1 & $-0,0628$ & 11 & 50,9 & $-0,0019$ & 356 & 69 & 0,975 \\
\hline & 6 & 55,9 & $-0,0653$ & 11 & 43,2 & $-0,0018$ & 378 & 32 & 0,983 \\
\hline & 9 & 55,2 & $-0,0460$ & 15 & 44,8 & $-0,0026$ & 269 & 45 & 0,979 \\
\hline & Média & 53,7 a & $-0,0580$ a & $12 \mathrm{a}$ & 46,3 c & $-0,0021 \mathrm{a}$ & $335 \mathrm{a}$ & $49 \mathrm{c}$ & \\
\hline CV\% & & 15,6 & 24,8 & 23,2 & 9,03 & 20,8 & 16,7 & 25,7 & \\
\hline
\end{tabular}

${ }^{1} \mathrm{NR}=$ Nível de resíduos (Mg ha ${ }^{-1}$ de MS). Os níveis de resíduos não foram estatisticamente diferentes.

${ }^{2}$ Médias não seguidas pela mesma letra minúsculas na coluna diferem pelo teste de Duncan $(\mathrm{P}<0,05)$.

Ciência Rural, v.44, n.5, mai, 2014. 

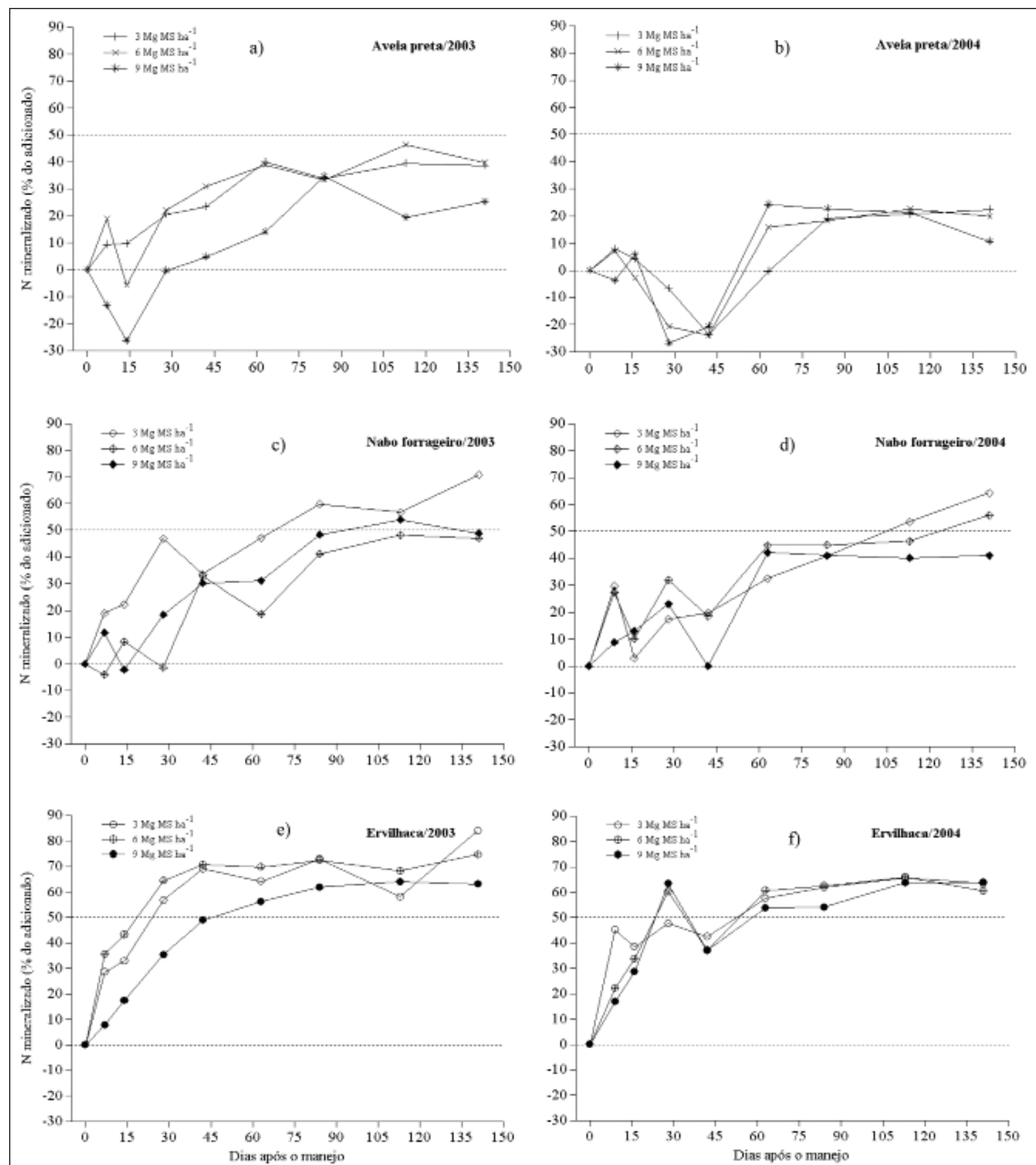

Figura 3- Percentual mineralizado do N adicionado ao solo com resíduos culturais de aveia preta, nabo forrageiro e ervilhaca em função da quantidade aportada nos sacos de decomposição.

uma liberação de N mais lenta do que em 2004. Já com os resíduos da aveia preta, em 2004, foi observada acentuada imobilização inicial (primeiros 50 dias). No entanto, em 2003, a imobilização foi menos intensa e de menor duração, ocorrendo apenas no tratamento com maior aporte de resíduos. Assim, em ambos os anos avaliados, o aporte de 9Mg ha ${ }^{-1}$ de MS promoveu imobilização temporária de até $30 \mathrm{~kg} \mathrm{ha}^{-1}$ de $\mathrm{N}$, aproximadamente, 15 e 30 dias após o manejo, para 2003 e 2004, respectivamente (Figura 3a e 3b). A remineralização parcial da quantidade imobilizada só ocorreu a partir de 30 e 60 dias após o 
manejo, para 2003 e 2004, respectivamente (Figura 3a e 3b). Assim, a imobilização e a remineralização de $\mathrm{N}$ por resíduos de aveia foi influenciada pelo ano de avaliação e pela quantidade de resíduos aportados. Segundo VARGAS et al. (2005), o nível mais elevado de carbono disponível para a biomassa microbiana no SPD possibilitaria uma maior imobilização do N, bem como o seu acúmulo gradual em formas orgânicas, aumentando a capacidade de suprimento deste nutriente ao longo do tempo. A dinâmica da liberação de $\mathrm{N}$ da aveia preta, observada neste experimento, evidenciou o processo de imobilização temporária com liberação parcial e tardia do nutriente (AMADO et al., 2003), como observado anteriormente por SÁ (1996) em estudo com antecipação de adubação nitrogenada no milho em sucessão à aveia. Os resíduos de nabo forrageiro apresentaram, quanto à liberação de $\mathrm{N}$, um comportamento intermediário entre a aveia preta (com imobilização temporária) e a ervilhaca (rápida mineralização).

O processo de decomposição dos resíduos culturais também foi avaliado por meio das constantes de decomposição $\left(\mathrm{k}_{\mathrm{a}}\right.$ e $\left.\mathrm{k}_{\mathrm{b}}\right)$ e o tempo de meia-vida $\left(t_{1 / 2}\right)$ dos resíduos vegetais subdivididos através do modelo duplo exponencial em compartimentos lábil e recalcitrante (Tabela 1). O compartimento lábil é a parte mais facilmente decomponível da fitomassa (AITA \& GIACOMINI, 2003) e os resíduos com maior magnitude deste compartimento apresentaram maior taxa de decomposição. Já o compartimento recalcitrante é formado por compostos orgânicos de mais difícil decomposição, como, por exemplo, lignina e polifenóis (PAUL \& CLARK, 2007) e condicionam uma menor taxa de decomposição (MEDRADO et al., 2011).

Os níveis de resíduos aportados não apresentaram efeito significativo sobre a magnitude dos compartimentos e o tempo de meia-vida (Tabela 1). Entretanto, os tipos de resíduos diferiram quanto à magnitude dos compartimentos lábil e recalcitrante e quanto ao tempo para decompor a metade dos resíduos aportados. Assim, a ervilhaca e o nabo forrageiro apresentaram compartimento lábil praticamente duas vezes superior ao da aveia preta. Por outro lado, a magnitude do compartimento recalcitrante seguiu a ordem: aveia preta, nabo forrageiro e ervilhaca. Na média dos níveis de resíduos investigados, a metade dos resíduos foram decompostos em 1,6, 3,5 e 5,5 meses para a ervilhaca, nabo forrageiro e aveia preta, respectivamente. Anteriormente, CERETTA et al. (2002) reportaram que, para decompor a metade dos resíduos de nabo forrageiro, seriam necessários aproximadamente 2 meses; enquanto CARVALHO et al. (2008) reportaram 3 meses, à semelhança deste trabalho. Para a aveia preta, TORRES \& PEREIRA (2008) reportaram o tempo de 3,7 meses, valor inferior ao observado neste trabalho (Tabela 1 ).

Os resíduos de aveia preta com, aproximadamente, $\quad 80 \%$ de compartimento recalcitrante necessitaram de 164 dias para decompor a metade da quantidade de seus resíduos, enquanto que a ervilhaca, com menos de $50 \%$ deste compartimento, levou apenas 49 dias (Tabela 1). Os resíduos de nabo forrageiro, com 60\% deste compartimento, necessitaram de 106 dias. No entanto, o tempo de meia-vida tanto do compartimento lábil como do recalcitrante não diferiu entre as espécies (Tabela 1). Esses resultados sugerem que a cinética de decomposição está intimamente associada ao tamanho de cada compartimento e não, necessariamente, às constantes de decomposição, que são variáveis em função das condições climáticas, como observado anteriormente por ESPÍNDOLA et al. (2006); TORRES et al. (2008).

A relação $\mathrm{C} / \mathrm{N}$ tem sido apontada por muitos trabalhos como principal parâmetro no processo de decomposição. Neste trabalho, associando-se a relação $\mathrm{C} / \mathrm{N}$ com a magnitude dos compartimentos, foi possível constatar que o aumento da relação $\mathrm{C} / \mathrm{N}$ está diretamente associado com o incremento do compartimento recalcitrante e inversamente com o compartimento lábil (Figura 4), justificando a importância da avaliação destes parâmetros em estudos de decomposição dos resíduos vegetais.

\section{CONCLUSÃO}

No sistema plantio direto, a taxa de decomposição não foi influenciada pela quantidade de resíduos aportados a superfície do solo, independente do tipo de resíduo investigado. Esse resultado foi atribuído à capacidade dos microrganismos em se adaptarem à maior oferta de $\mathrm{C}$, sob condições não limitantes de temperatura e umidade. Entretanto, os processos de mineralização e imobilização de $\mathrm{N}$ foram condicionados pelo nível de resíduo aportado. Ambos os processos, decomposição e liberação de $\mathrm{N}$, foram regulados pela relação $\mathrm{C} / \mathrm{N}$ da fitomassa das plantas de cobertura, seguindo a ordem: ervilhaca, nabo forrageiro e aveia preta. O incremento da relação $\mathrm{C} / \mathrm{N}$ da fitomassa relacionou-se inversamente com 


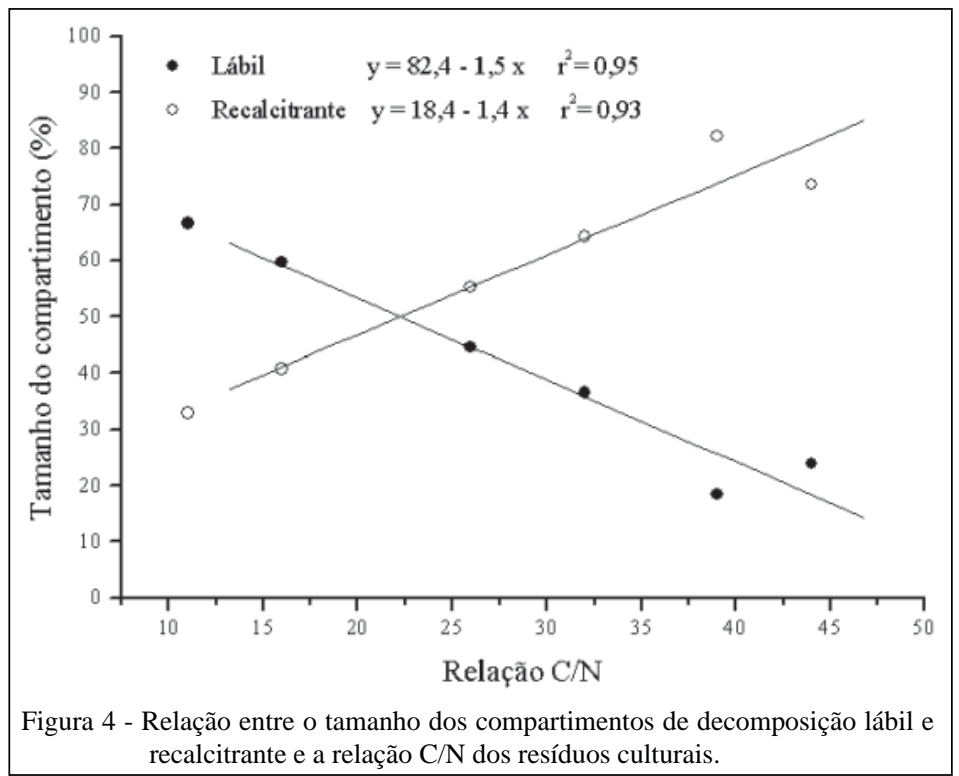

a magnitude do compartimento lábil e diretamente com o do recalcitrante. Esse fato foi determinante para o decréscimo da taxa de decomposição e da liberação de $\mathrm{N}$ da fitomassa das plantas de cobertura.

\section{AGRADECIMENTOS}

Ao Conselho Nacional de Desenvolvimento Científico e Tecnológico (CNPq) pela bolsa de pesquisa ao segundo autor e financiamento através dos projetos MCT/CNPq 14/2010 480162/2010-7 and MCT/CNPq 14/2012 - 485896/2012-5.

\section{REFERÊNCIAS}

ACOSTA, J.A.A. et al. Effect of ${ }^{15} \mathrm{~N}$-labeled hairy vetch and nitrogen fertilization on maize nutrition and yield under no-tillage. Revista Brasileira de Ciência do Solo, v.35, n.4, p.1337-1345, 2011. Disponível em: <http://www.scielo.br/pdf/rbcs/v35n4/a28v35n4.pdf>. Acesso em: 8 jun. 2013. doi: 10.1590/S0100-06832011000400028.

AITA, C. Dinâmica do nitrogênio no solo durante a decomposição de plantas de cobertura: efeito sobre a disponibilidade de nitrogênio para a cultura em sucessão. In: FRIES, M.R.; DALMOLIN, R.S.D. Atualização em recomendação de adubação e calagem: ênfase em plantio direto. Santa Maria: Depto de Solos/UFSM, 1997. p.76-111.

AITA, C.; GIACOMINI, S.J. Decomposição e liberação de nitrogênio de resíduos culturais de plantas de cobertura de solo solteiras e consorciadas. Revista Brasileira de Ciência do Solo, v.27, n.4 p.601-612, 2003.Disponível em: <http://www.scielo. br/pdf/rbcs/v27n4/a04v27n4.pdf>. Acesso em: 7 jan. 2014. doi: 10.1590/S0100-06832003000400004.

AMADO, T.J.C. et al. Flutuação de temperatura e umidade do solo sob preparo convencional e em faixas na cultura da cobertura. Pesquisa Agropecuária Brasileira, v.25, p.625-631, 1990.

AMADO, T.J.C. et al. Leguminosas e adubação mineral como fontes de nitrogênio para o milho em sistemas de preparo do solo. Revista Brasileira de Ciência do Solo, v.24, p.179-189, 2000.
Disponível em: <http://sbcs.solos.ufv.br/solos/revistas/v24n1a20. pdf $>$. Acesso em: 8 jan. 2014.

AMADO, T.J.C. et al. Recomendação de adubação nitrogenada para o milho no RS e SC adaptado ao uso de culturas de cobertura, sob sistema plantio direto. Revista Brasileira de Ciência do Solo, v.26, p.241-248, 2002.Disponível em: <http://sbcs.solos.ufv.br/ solos/revistas/v26n1a25.pdf>. Acesso em: 10 set. 2013.

AMADO, T.J.C. et al. Adubação nitrogenada na aveia preta: influência na decomposição de resíduos, liberação de nitrogênio e rendimento de milho sob sistema plantio direto. Revista Brasileira de Ciência do Solo, v.27, p.1085-1096, 2003.Disponível em: $<$ http://www.redalyc.org/articulo.oa?id=180214033012 >. Acesso em: 8 jan. 2014. doi: 10.1590/S0100-06832003000600013.

AMADO, T.J.C. et al. Potential of Carbon Accumulation in No-Till Soils with Intensive Use and Cover Crops in Southern Brazil. Journal of Environmental Quality, v.35, p. 15991607, 2006. Disponível em <https://dl.sciencesocieties.org/ publications/jeq/abstracts/35/4/1599>. Acesso em: 6 jun. 2013. doi:10.2134/jeq2005.0233.

CARVALHO, A.M.et al. Decomposição de resíduos vegetais em latossolo sob cultivo de milho e plantas de cobertura. Revista Brasileira de Ciência do Solo, v.32, p.2831-2838, 2008. Disponível em: <http://www.scielo.br/scielo.php?pid=S010006832008000700029\&script=sci_arttext $>$. Acesso em: 8 jan. 2014. doi: 10.1590/S0100-06832008000700029.

CERETTA, C.A. et al. Produção e decomposição de fitomassa de plantas invernais de cobertura de solo e milho, sob diferentes manejos da adubação nitrogenada. Ciência Rural, v.32, p.49-54, 2002. Disponível em: <http://www.scielo.br/scielo. php?pid=S0103-84782002000100009\&script=sci_arttext $>$. Acesso em: 8 jan. 2014. doi:10.1590/S0103-84782002000100009.

DERPSCH, R. et al. Current status of adoption of no-till farming in the world and some of its main benefits. Journal Agriculture \& Biology, v.3, p.1-25, 2010. Disponível em: <http://www.ijabe.org/ index.php/ijabe/article/view/223>. Acesso em: 3 jun. 2013. doi: 10.3965/j.issn.1934-6344.2010.01.0-0. 
EMPRESA BRASILEIRA DE PESQUISA AGROPECUÁRIA (EMBRAPA). Sistema Brasileiro de Classificação de Solos. 2.ed. Rio de Janeiro, 2006. 412p.

ESPÍNDOLA, J.A.A. et al. Decomposição e liberação de nutrientes acumulados em leguminosas herbáceas perenes consorciadas com bananeira.Revista Brasileira de Ciência do Solo, v.30, p.321328, 2006.Disponível em: <http://www.scielo.br/pdf/rbcs/v30n2/ a12v30n2.pdf > . Acesso em: 8 jan. 2014. doi: 10.1590/S010006832006000200012.

JANSSEN, B.H. Nitrogen mineralization in relation to C:N ratio and decomposability of organic materials. Plant and Soil, v.181, p.39-45, 1996. Disponível em: <http://link.springer.com/ article/10.1007/BF00011290>. Acesso em: 3 jan. 2013. doi: 10.1023/A:1004336903214.

JENSEN, E.S. Nitrogen immobilization and mineralization during initial decomposition of ${ }^{15} \mathrm{~N}$-labelled pea and barley residues. Biology and Fertility of Soils, v.24, p.39-44, 1997. Disponível em:<http://link.springer.com/article/10.1007/BF01420218>. Acesso em: 5 jan. 2013. doi: 10.1007/BF01420218.

KOENIG, R.T.; COCHRAN, V.L. Decomposition and nitrogen mineralization from legume and non-legume crop residues in a subarctic agricultural soil. Biology and Fertility of Soils, v.17, p.269-275, 1994. Disponível em: <http://link.springer.com/ article/10.1007/BF00383980>. Acesso em: 5 jun. 2013. doi: 10.1007/BF00383980.

KUDEYAROV, V.N. The nitrogen and carbon balance in soil. Eurasian Soil Science, v.32, p.73-82, 1999.

KUMAR, K.; GOH, K.M. Nitrogen release from crop residues and organic amendments as affected by biochemical composition. Communications in Soil Science and Plant Analysis, v.34, p. 2441-2460, 2003. Disponível em: <http://www.tandfonline.com/ doi/abs/10.1081/CSS-120024778\#.Us586fRDve4>. Acesso em: 5 jun. 2013. doi: 10.1081/CSS-120024778.

MARY, B. et al. Interaction between decomposition of plant residues and nitrogen and nitrogen cycling in soil. PlantandSoil, v.181, p.71-82, 1996. Disponível em: <http://link.springer.com/ article/10.1007/BF00011294>.Acesso em: 5 jun. 2013 . doi: 10.1007/BF00011294.

MEDRADO, R.D. et al. Decomposição de resíduos culturais e liberação de nitrogênio para a cultura do milho. Scientia Agrária, v.12, p.97-107, 2011. Disponível em: <http://ojs.c3sl.ufpr.br/ojs2/index.php/agraria/ article/view/33761/21118>. Acesso em: 12 jun. 2013.
MONTEIRO, H.C.F. et al. Dinâmica de decomposição e mineralização de nitrogênio em função da qualidade de resíduos de gramíneas e leguminosas forrageiras. Revista Brasileira de Zootecnia, v.31, p.1092-1102, 2002. Disponível em: <http://www. scielo.br/pdf/rbz/v31n3/13060.pdf>. Acesso em: 15 jun. 2013. doi: 10.1590/S1516-35982002000500005.

PARTON, W. et al. Global-scale similarities in nitrogen release patterns during long-term decomposition. Science, v.315, p.361364, 2007. Disponível em: <http://www.ncbi.nlm.nih.gov/ pubmed/17234944>. Acesso em: 5 jun. 2013. doi:10.1126/ science. 1134853 .

PAUL, E.A.; CLARK, F.E. Soil microbiology and biochemistry. 3.ed. California: Academic, 2007. 340p.

PLANTAE, A.F.; PARTON, W.J. The dynamics of soil organic matter and nutrient cycling.In: PAUL, E. Soil microbiology, ecology, and biochemistry. 3.ed. California: Academic, 2007. p.433-464.

ROBERTSON, G.P.; GROFFMAN, P.M. Nitrogen transformations. In: PAUL, E. Soil microbiology, ecology, andbiochemistry.3.ed. California: Academic, 2007. p.341-264.

SÁ, J.C.M. Manejo de nitrogênio na cultura de milho no sistema plantio direto. Passo Fundo: Aldeia Norte, 1996. 23p.

SAS Institute. SAS user's guide: statistic.Cary, 1990.846p.

TEDESCO, M.J. et al. Análise de solo, plantas e outros materiais. 2.ed. Porto Alegre: Universidade Federal do Rio Grande do Sul, 1995. 174p.

TORRES, J.L.R.; PEREIRA, M.G. Dinâmica do potássio nos resíduos vegetais de plantas de cobertura no cerrado. Revista Brasileira de Ciência do Solo, v.32, p.1609-1618, 2008. Disponível em: <http://www.scielo.br/pdf/rbcs/v32n4/ a25v32n4.pdf $>$. Acesso em: 12 jun. 2013. doi: 10.1590/S010006832008000400025.

TORRES, J.L.R. et al. Produção de fitomassa por plantas de cobertura e mineralização de seus resíduos em plantio direto. Pesquisa Agropecuária Brasileira, v.43, p. 421-428, 2008. Disponível em: <http://www.scielo.br/pdf/pab/v43n3/ a18v43n3.pdf $>$. Acesso em: 5 jan. 2013. doi: 10.1590/S0100204X2008000300018.

VARGAS, L.K. et al. Imobilização de nitrogênio em solo cultivado com milho em sucessão à aveia preta nos sistemas plantio direto e convencional. Ciência Rural, v.35, p.76-83, 2005. Disponível em: $<$ http://www.scielo.br/pdf/cr/v35n1/a12v35n1.pdf>. Acesso em: 10 mai. 2013. doi: 10.1590/S0103-84782005000100012. 